\title{
Anisotropy of the Charge Transport in $\mathrm{GdB}_{6}$
}

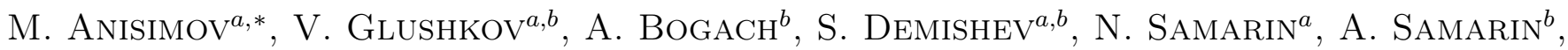 \\ N. Shitsevalova ${ }^{c}$, A. Levehenko $^{c}$, V. Filipov $^{c}$, S. GÁbani $^{d}$, K. Flachbart $^{d}$ \\ AND N. SLuCHANKO ${ }^{a, b}$
}

${ }^{a}$ Prokhorov General Physics Institute of RAS, Vavilov 38, 119991 Moscow, Russia

${ }^{b}$ Moscow Institute of Physics and Technology, Institutsky lane 9, 141700 Dolgoprudny, Russia

${ }^{c}$ Frantsevich Institute for Problems of Materials Science NAS, Krzhyzhanovskii 3, 03680 Kiev, Ukraine

${ }^{d}$ Institute of Experimental Physics of SAS, Watsonova 47, SK-04001 Košice, Slovak Republic

The anisotropy of charge transport was investigated in the antiferromagnetic II state of $\mathrm{GdB}_{6}$ from precise measurements of transverse magnetoresistance. Based on the data obtained we detected a complicated behavior of magnetoresistance curves which are characterized by the appearance of considerable hysteresis on the field and angular dependences below $T_{N 2}$. Moreover it was shown that the system $\mathrm{GdB}_{6}$ is sensitive to coolingwarming prehistory. The data analysis allowed to reconstruct magnetic $H-T$ phase diagram of $\mathrm{GdB}_{6}$ along main crystallographic directions $(\boldsymbol{H} \|\langle 001\rangle,\langle 110\rangle,\langle 111\rangle)$ and to propose additional phase transition inside AF II phase at $H_{1} \approx 0.5 \mathrm{~T}$.

DOI: 10.12693/APhysPolA.131.973

PACS/topics: $72.15 . \mathrm{Gd}$

\section{Introduction}

Gadolinium hexaboride $\left(\mathrm{GdB}_{6}\right)$ is the $S$-state system (for $\mathrm{Gd}^{3+}, L=0, S=7 / 2$ ) which represents very unusual physical properties [1-14]. Similar to another rare earth (RE) hexaborides $\mathrm{GdB}_{6}$ crystallizes in a simple bcc structure of $\mathrm{CsCl}$ type (s.g. $P m 3 m-O_{h}^{1}$ ) and shows the flat phonon dispersion relations around $10 \mathrm{meV}$ [15] corresponding to the motion of $\mathrm{RE}$ ions inside a rigid boron cage. However in $\mathrm{GdB}_{6}$ the amplitude of thermal vibrations of Gd ions is much larger in comparison with other $\mathrm{RE}$ ions in $\mathrm{RB}_{6}$ family [16]. $\mathrm{GdB}_{6}$ demonstrates two successive first-order antiferromagnetic (AF) transitions with simultaneous structural distortions to AF I [or $\mathrm{H}]$ state at $T_{N 1} \approx 15 \mathrm{~K}$ and to AF II [or L] state below $T_{N 2}$. (The value of $T_{N 2}$ is found to be strongly sample dependent ranging in the interval $T_{N 2} \approx 5-10 \mathrm{~K}$ [1-14]). Recent structural investigations (including X-ray [8-12] and neutron diffraction $[13,14]$ experiments) have shown that commensurate magnetic structure with the wave vector $\boldsymbol{k}_{m}=[1 / 4,1 / 4,1 / 2]$ is realized both in AF I and AF II states of $\mathrm{GdB}_{6}$. The changes between AF I and AF II phases are connected with appearance of different reflexes corresponding to the structural modulation. According to the last X-ray experiments $[11,12]$ lattice distortions with $\boldsymbol{q}_{1}=[1 / 2,0,0]$ and $\boldsymbol{q}_{2}=[1 / 2,1 / 2,0]$ vectors were observed in AF I state whereas the same reflexes $\boldsymbol{q}_{1}, \boldsymbol{q}_{2}$ with additional one $\boldsymbol{q}_{3}=[1 / 4,1 / 4,1 / 2]$ (identical to the magnetic wave vector $\boldsymbol{k}_{m}$ ) were detected in AF II phase below $T_{N 2}$. (The description of modulated structures in literature often contains the results of previous X-ray studies [8-10] with $\boldsymbol{q}_{1}$ vector in AF I state and $\boldsymbol{q}_{2}$ vector in AF II phase.)

\footnotetext{
*corresponding author; e-mail: anisimov.m.a@gmail.com
}

Theoretical models presented for $\mathrm{GdB}_{6}$ in literature $[11,17]$ consider a coherent displacement of Gd ions inside the rigid boron cage. In particular, Kasuya [17] applied the Jahn-Teller formalism to $\mathrm{RB}_{6}$ compounds and proposed a stable coexistence of charge dipole and magnetic dipole ordering in both $\mathrm{AF}$ states of $\mathrm{GdB}_{6}$. However, the magnetic dipole ordering of Gd-Gd pairs does not correspond to the vector of magnetic structure $\boldsymbol{k}_{m}$ obtained later from neutron studies $[13,14]$. Modern theory was proposed by Amara et al. in [11] for AF I state of $\mathrm{GdB}_{6}$. They introduced a mean-field model which predicts the coexistence of magnetic and displacement waves starting from isotropic exchange. This scenario [11] allowed to describe both the magnetic structure with $\boldsymbol{k}_{m}$ and structural modulated reflexes $\boldsymbol{q}_{1}$ and $\boldsymbol{q}_{2}$ detected in AF I state in $[11,12]$. However for AF II state the authors [11] mentioned that the displacement structure below $T_{N 2}$ cannot be identified in terms of their formalism. In this respect, a detailed investigation of the transport properties especially in the AF II state will provide additional information to unravel the mechanisms leading to the observed magnetic ground state $\mathrm{GdB}_{6}$.

\section{Experimental details}

The high quality $\mathrm{GdB}_{6}$ single crystal $\left(\rho_{300 \mathrm{~K}} / \rho_{2 \mathrm{~K}} \approx 6\right.$, $\left.T_{N 1} \approx 15.5 \mathrm{~K}, T_{N 2} \approx 4.7 \mathrm{~K}\right)$ was grown by vertical crucible-free inductive zone melting in argon gas atmosphere. The crystal quality was controlled by X-ray diffraction, the Laue backscattering patterns and electron microprobe analysis. Transverse magnetoresistance (MR) was measured in the temperature range $2-5 \mathrm{~K}$ in magnetic field up to $8 \mathrm{~T}$ by stepwise sample rotation technique. The step motor allowed a step-by-step rotating of sample $\left(\Delta \varphi \approx 1.8^{\circ}\right)$ in fixed magnetic field. In rotating experiment dc-current was applied along $\langle 110\rangle$ axis, whereas the vector of magnetic field $\boldsymbol{H}$ gradually passed $-180^{\circ} \div 180^{\circ}$ interval including main crystallographic directions (see Fig. 1a). In this study the special attention 
was paid for a high accuracy of temperature stabilization $(\Delta T \approx 0.001 \mathrm{~K}$ at $4 \mathrm{~K})$ which was achieved by using a modified commercial temperature controller TC1.5/300 (CRYOTEL Ltd.) in combination with temperature sensors CERNOX-1050 (Lake Shore Cryotronics Inc.). The originally designed experimental setup was described in detail in [18].
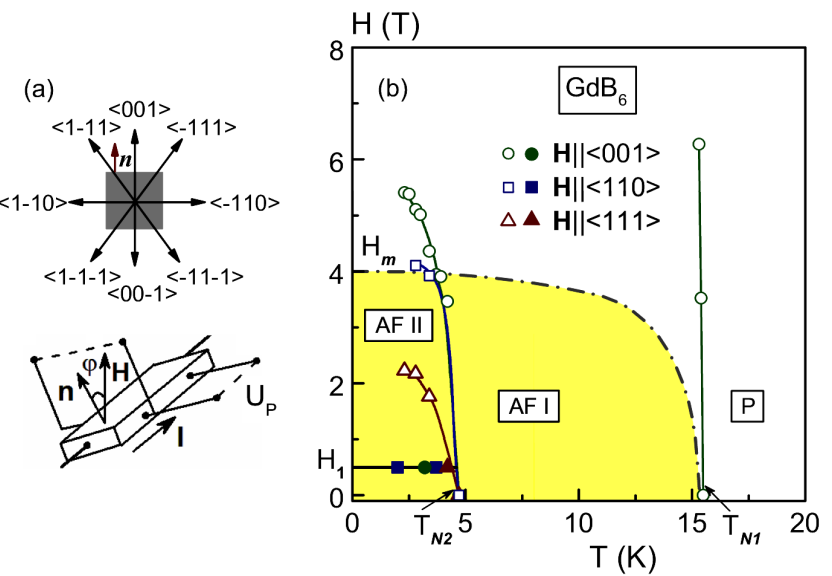

Fig. 1. (a) Experimental geometry of experiment with sample rotation. (b) Magnetic $H-T$ phase diagram of $\mathrm{GdB}_{6}$ for main crystallographic directions $\boldsymbol{H} \|\langle 001\rangle$, $\langle 110\rangle$ and $\langle 111\rangle$. A contour area $\left(H<H_{m}\right)$ represents the range with magnetic memory effects (see the text).

\section{Results and discussion}

1. We start the presentation of our data by considering the field dependences of transverse MR $\Delta \rho(H) / \rho$ in AF II state of $\mathrm{GdB}_{6}$ (Fig. 2) measured (a) at various temperatures in the range $2 \div 4.2 \mathrm{~K}$ for $\boldsymbol{H} \|\langle 110\rangle$ and (b) at $T_{0}=3.4 \mathrm{~K}$ for three main crystallographic directions. In spite of the obvious difference of the amplitude, the MR data for each crystallographic direction are characterized by a complex behavior including the crossover from positive $(\Delta \rho / \rho>0)$ to negative $(\Delta \rho / \rho<0)$ regime (Fig. 2a,b). The main fingerprint of AF II phase of $\mathrm{GdB}_{6}$ is the appearance of thermal, field and angular hysteresis on the transport and magnetic characteristics below $T_{N 2}$ (see Fig. 2 and also $[1-3,6]$ ). Moreover it is worth noting that $\mathrm{GdB}_{6}$ is very sensitive to cooling-warming prehistory in AF state (this was especially pointed by several groups of authors [1-3]). In particular strong influence of cooling-heating process on MR data may be illustrated for the case $T_{0}=4.2 \mathrm{~K}$ (Fig. 2a). Indeed, two types of field hysteresis $\left(A_{i}=\Delta \rho\left(H_{i+}\right) / \rho-\Delta \rho\left(H_{i-}\right) / \rho\right.$, where $i=1,2)$ were observed in the experiments with various temperature prehistories. The first $\left(A_{1}<0\right.$, see the solid line in Fig. 2a) was measured after rapid warming from low temperatures $2 \div 3 \mathrm{~K}$ within the first sweep of magnetic field up to the value $H_{m} \approx 4 \mathrm{~T}$ which evidently depresses the memory effects. The second $\left(A_{2}>0\right.$, the solid symbols on Fig. 2a) was obtained after stepwise warming from low to high temperatures. Further cycling of magnetic field totally reproduces the curve 2 . The unique way to reproduce the behavior of the curve 1 is to repeat rapid warming from low temperatures. This effect was also detected within the first scan of magnetic field after rapid cooling of the sample from high temperatures.
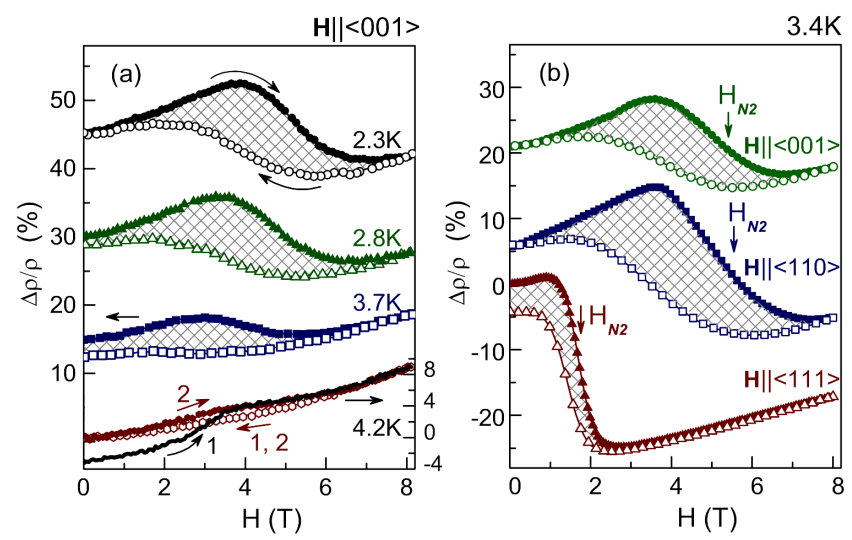

Fig. 2. Field dependences of transverse MR $\Delta \rho(H) / \rho$ in AF II state of $\mathrm{GdB}_{6}$ (a) at various temperatures in the range $2 \div 4.2 \mathrm{~K}$ for $\boldsymbol{H} \|\langle 001\rangle$ and (b) at $T_{0}=3.4 \mathrm{~K}$ for three main crystallographic directions. The curves are shifted for convenience. The numbers in the part (a) correspond to the numbers of scans of magnetic field at $T_{0}=4.2 \mathrm{~K}$ (see the text). Arrows denote AF (II)-AF (I) transition.

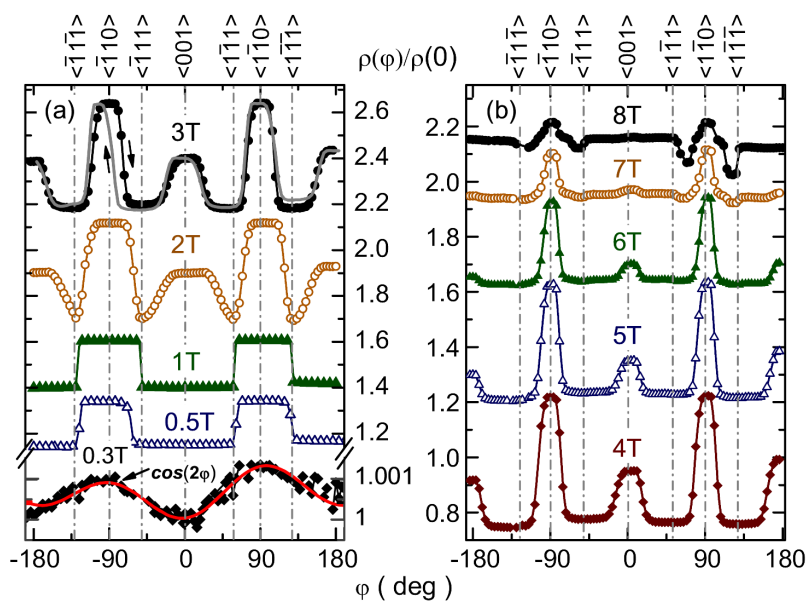

Fig. 3. Angular dependences of normalized MR $\rho\left(\varphi, T_{0}, H_{0}\right) / \rho\left(\varphi=0^{\circ}\right)$ in AF II state of $\mathrm{GdB}_{6}$ measured at $T_{0}=2 \mathrm{~K}$ for the various magnetic fields in the range $0.3 \div 8 \mathrm{~T}$. The curves are shifted for convenience.

2. In order to estimate the effects of magnetic anisotropy the angular dependences of normalized MR were investigated in AF II state of $\mathrm{GdB}_{6}$. Two isotherms $T_{0}=2 \mathrm{~K}$ and $T_{0}=3.4 \mathrm{~K}$ were studied at various magnetic fields in the range $0.3 \div 8 \mathrm{~T}$. The data obtained show the same behavior therefore we discuss here only the results for $T_{0}=2 \mathrm{~K}$. In order to avoid angular hysteresis effect the measurements were performed for both directions of rotation $\left(\Delta \varphi_{+}\right.$and $\left.\Delta \varphi_{-}\right)$. Figure 3a,b presents the re- 
sultant normalized $\rho\left(\varphi, H_{0}, T_{0}\right) / \rho\left(\varphi=0^{\circ}\right)$ data shifted along ordinate axis for convenience. It is evident that the character of transport anisotropy changes drastically (i)-(iii) with magnetic field.

(i) Indeed, in the range $H<H_{1} \approx 0.5 \mathrm{~T}$ the $\rho(\varphi) / \rho(0)$ dependences of a low amplitude can be approximated by the relation $\rho(\varphi) \approx \cos (2 \varphi)$ which is typical for the weakly anisotropic crystals of the cubic symmetry.

(ii) In the following interval of magnetic fields $0.5-H_{2} \approx 1.7 \mathrm{~T}$ the MR curves of a noticeable amplitude $(>7 \%)$ take the shape of meander with extended plateau $\rho(\varphi) / \rho(0)=$ const which are followed by a periodic switching at angles in the narrow vicinity of $\boldsymbol{H} \|\langle 111\rangle$ directions (a width smaller than $5^{\circ}$ ), Fig. 3a.

(iii) Starting from point $H_{2} \approx 1.7 \mathrm{~T}$ the shape of $\rho(\varphi) / \rho(0)$ curves changes again representing the caverns (or beak-shaped minimums) along the four $\boldsymbol{H} \|\langle 111\rangle$ directions in combination with peaks of maximal scattering along $\boldsymbol{H} \|\langle 110\rangle$ and $\boldsymbol{H} \|\langle 001\rangle$. The maximum of resistivity ratio $\rho(\boldsymbol{H} \|\langle 110\rangle) / \rho(\boldsymbol{H} \|\langle 111\rangle) \sim 1.57$ is achieved at $H \sim 4 \mathrm{~T}$.

3. The data obtained allow us to reconstruct magnetic $H-T$ phase diagram for main crystallographic directions $\boldsymbol{H} \|\langle 001\rangle,\langle 110\rangle,\langle 111\rangle$ in AF II state of $\mathrm{GdB}_{6}$ (Fig. 1b). Taking into account the detected peculiarities on the field $\Delta \rho(H) / \rho$ dependences we estimated the position of the phase boundary $T_{N 2}(H)$ from the inflection points (see the arrows in Fig. 2b). $T_{N 2}(H)$ branches obtained in current study (open symbols in Fig. 1b) reproduces in good agreement the characteristic view of $\mathrm{AF}(\mathrm{I})-\mathrm{AF}(\mathrm{II})$ phase boundary reported previously in $[7,12]$ including a low-type behavior of $T_{N 2}^{111}(H)$ curve. Moreover, our results allow us to propose at least one additional transition inside AF II state at $H_{1} \approx 0.5 \mathrm{~T}$ for all applied field directions (see solid symbols in Fig. 1b). This transition which phase boundary $H_{1}(T)$ has nearly horizontal view was observed previously in the range $H_{1} \approx 0.4 \div 0.7 \mathrm{~T}$ from magnetization and torque measurements $[2,3,5]$ performed on single(poly-) crystals of $\mathrm{GdB}_{6}$ of a various quality. Besides the character of $H_{1}(T)$ curve probably corresponds to some field induced magnetization processes. Another one point $H_{2} \approx 1.7 \mathrm{~T}$ representing the appearance of the caverns along four $\boldsymbol{H} \|\langle 111\rangle$ directions (see $H_{0}=2 \mathrm{~T}$ in Fig $3 \mathrm{a}$ ) should be ascribed to the phase boundary $T_{N 2}^{111}(H)$. There is no any contradiction as it seems at the first sight, since the strong hysteresis effect which provides the considerable difference $\Delta H \approx 0.5 \mathrm{~T}$ between the results in field and rotating experiments. We also designate the range of magnetic fields $\left(H<H_{m} \approx 4 \mathrm{~T}\right)$ where the magnetic memory effects [including the influence of the temperature prehistory] were detected. A detailed analysis of magnetic $H-T$ phase diagram for both $\mathrm{AF}$ states of $\mathrm{GdB}_{6}$ will be presented elsewhere. We believe that the study of $S$-system $\mathrm{GdB}_{6}$ will help to separate effects produced by spin and orbital degrees of freedom and to clarify their role on the mechanisms responsible for the ground state formation in all $\mathrm{RB}_{6}$ metallic antiferromagnets.

\section{Conclusions}

We have performed the precision measurements of MR in AF II state of $\mathrm{GdB}_{6}$. Our MR data demonstrate the complicated behavior with appearance of considerable hysteresis on the field and angular dependences below $T_{N 2}$. The analysis of the results obtained allowed us to reconstruct magnetic $H-T$ phase diagram of $\mathrm{GdB}_{6}$ along the main crystallographic directions and to propose additional phase transition inside AF II state at $H_{1} \approx 0.5 \mathrm{~T}$.

\section{Acknowledgments}

This work was supported by the Department of Physical Sciences of RAS (program Strongly Correlated Electrons in Metals, Semiconductors, and Magnetic Materials), Slovak Scientific Grant Agencies VEGA (2/0106/13) and APVV (14-0605).

\section{References}

[1] S. Kunii, K. Takeuchi, I. Oguro, K. Sogiyama, A. Ohya, M. Yamada, Y. Koyoshi, M. Date, T. Kasuya, J. Magn. Magn. Mater. 52, 275 (1985).

[2] N. Ali, J. Appl. Phys. 63, 3583 (1988).

[3] H. Nazaki, T. Tanaka, Y. Ishizawa, J. Phys. C Solid State Phys. 13, 2751 (1980).

[4] M. Anisimov, A. Bogach, V. Glushkov, S. Demishev, N. Samarin, N. Shitsevalova, A. Levchenko, V. Filipov, A. Kuznetsov, N. Sluchanko, J. Phys. Conf. Ser. 400, 032003 (2012).

[5] H. Hacker, Y. Shimada, K. Chung, Phys. Status Solidi A 4, 459 (1971).

[6] M. Reiffers, J. Šebek, E. Šantavá, G. Pristáš, S. Kunii, Phys. Status Solidi B 243, 313 (2006).

[7] R.-M. Galéra, P. Morin, S. Kunii, T. Kasuya, J. Magn. Magn. Mater. 104-107, 1336 (1992).

[8] R.-M. Galéra, D.P. Osterman, J.D. Axe, J. Appl. Phys. 63, 3580 (1988).

[9] K. Kuwahara, R. Yamamoto, M. Kohgi, H. Nakao, K. Ishii, K. Iwasa, Y. Murakami, S. Kunii, H. Sagayama, Y. Wakabayashi, H. Sawa, Physica B 359-361, 965 (2005).

[10] D.F. McMorrow, K.A. McEwen, J.-G. Park, S. Lee, D. Mannix, F. Iga, T. Takabatake, Physica B 345, 66 (2004).

[11] M. Amara, S.E. Luca, R.-M. Galéra, F. Givord, C. Detlefs, S. Kunii, Phys. Rev. B 72, 064447 (2005).

[12] S. Luca, Ph.D. thesis, Université Joseph-Fourier, Grenoble 2002.

[13] S. Luca, M. Amara, R.-M. Galéra, F. Givord, S. Granovsky, O. Isnard, B. Beuneu, Physica B 350, e39 (2004).

[14] K. Kuwahara, S. Sugiyama, K. Iwasa, M. Kohgi, M. Nakamura, S. Kunii, Appl. Phys. A 74, S302 (2002).

[15] N. Ogita, S. Nagai, N. Okamoto, M. Udagawa, F. Iga, M. Sera, J. Akimitsu, S. Kunii, Phys. Rev. B 68, 224305 (2003).

[16] Y. Takahashi, K. Ohshima, F. Okamura, S. Otani, T. Tanaka, J. Phys. Soc. Jpn. 68, 2304 (1999).

[17] T. Kasuya, J. Magn. Magn. Mater. 174, L28 (1997).

[18] I. Lobanova, V. Glushkov, N. Sluchanko, S. Demishev, Sci. Rep. 6, 22101 (2016). 